\title{
Redes de asociaciones migrantes y actores españoles: Un modelo de integración desde la capitalización social interorganizativa
}

\author{
José Manuel Gaete Fiscella \\ Universidad de Los Lagos, Santiago, Chile. \\ Email: josemanuel.gaete@ulagos.cl

\section{Paola Andrea Ilabaca Baeza} \\ Universidad Santo Tomás, Santiago, Chile. \\ Email: pilabaca@santotomas.cl
}

\begin{abstract}
Resumen $^{1}$ : Este artículo tiene como finalidad proponer y presentar un modelo de integración social a nivel organizacional de las asociaciones de migrantes extracomunitarios en España. Para esto se analizó el stock de capital social relacional que desarrollaron las asociaciones con actores del entorno, apuntando específicamente a la particularidad de las relaciones contraídas según la frecuencia de las mismas, el grado de orientación (actores nativos o migrantes) y, finalmente, el grado de simetría decisional en que se desarrollaron dichas relaciones. Los datos se extraen de una muestra representativa conformada por 225 asociaciones de migrantes de todo el territorio español. El análisis de la información permite definir un modelo de integración para estas organizaciones, caracterizando y discriminando con claridad las posibilidades de integración de las asociaciones, los tipos de capital social recreado y las posibilidades diferenciadas de integración que pueden evidenciar las asociaciones según su origen o nacionalidad.
\end{abstract} integración.

Palabras clave: Asociacionismo, migrante, capital social, redes sociales,

\section{Migrant associations networks and spanish actors: An integration model out of inter-organizational social capitalization}

\begin{abstract}
The aim of this article is to propose and present a model of social integration at the organizational level of EU migrants associations in Spain. To that end, it analyses the stock of relational capital developed by those associations and other actors of the social context, focusing specifically on the distinctive features of their interactions such and their frequency, the guidance level (native actors or migrants) and finally the degree of decisional symmetry within those relations. The data are drawn from a representative sample of 225 immigrant associations all over Spain. The data analysis allows defining a model of integration for these organizations, characterizing and clearly differencing the possibilities of integration of the associations, the types of social capital recreated and the influence of nationality
\end{abstract}


on the degree of integration of those associations.

Keywords: associations, immigrant, social capital, social networks, integration.

\title{
Redes de associações de migrantes e atores espanhóis: Um modelo de integração desde a capitalização social inter-organizacional
}

\begin{abstract}
Resumo: Este trabalho tem como objetivo propor e apresentar um modelo de integração social em nível organizacional das associações de migrantes extracomunitários na Espanha. Para isso, analisou-se o estoque de capital social relacional que desenvolveram as associações com atores do ambiente, apontando especificamente para a particularidade das relações contraídas de acordo com a frequência das mesmas, o grau de orientação (atores nativos ou imigrantes) e, finalmente, o grau de simetria decisória em que se desenvolveram tais relações. Os dados foram extraídos de uma amostra representativa composta por 225 associações de migrantes em todo o território espanhol. A análise dos dados permite definir um modelo de integração para estas organizações, caracterizar e discriminar claramente as possibilidades de integraçãodas associações, os tipos de capital social recriado e as possibilidades diferenciadas de integração que podem mostrar as associações de acordo com sua origem ou nacionalidade.
\end{abstract} integração.

Palavras-chave: associacionismo, migrantes, capital social,redes sociais, ***

\section{Introducción}

El trabajo que aquí se presenta busca aportar y complementar el estudio sobre el fenómeno migrante en general y latinoamericano en particular, prestando especial atención sobre el proceso de integración que alcanzan los colectivos extranjeros, desde un nivel agregado, en la sociedad de acogida española. Para esto nos centraremos en la expresión colectiva que suponen las asociaciones de migrantes y su relevancia en tanto mecanismo de integración para los mismos migrantes como de cohesión para los nativos (Jensen, 2010; Brazán, 2009). Es decir, la integración social migrante como una problemática que también se discute y analiza en las coordenadas mesosociales, y que se evidencia y constata a través de la participación del asociacionismo migrante en la sociedad de acogida (González y Morales, 2006).

Además de esta definición de nivel, la perspectiva que aquí se presenta busca ahondar en la integración social del migrante como sujeto, esto es, considerando su propia acción y agencia para participar en y de la sociedad de acogida (Solé y Perella, 2005; Del Olmo, 2003), lo que supone, por un lado, el estudio de las acciones y relaciones que los propios migrantes recrean y emprenden para la construcción de una sociedad civil migrante, en tanto sujeto activo y particular que, en la relación con otros no es sólo 
receptor de las contingencias del entorno y de su deriva migratoria, sino que desarrolla estrategias de diversa naturaleza que buscan su representación (más o menos participativa) en la sociedad de acogida a partir de la construcción de entidades organizacionales o asociaciones, y que, en su conjunto, dan cuenta de lo que se denomina una ciudadanía (Cinalli 2007; Toral, 2009) o comunidad migrante (Stefoni, 2004). Por otro lado, se hace necesario estudiar cómo estas asociaciones, en tanto construcciones activas, resultan ser sujetos migrantes colectivos que responden a un ethos migrante expresado en la emergencia y producto de la acción asociativa migrante, y también, por la funcionalidad que cumple para promover acciones orientadas a superar la condición migrante de sus representados, y por tanto, como un sujeto migrante mesosocial que también reclama su propio proceso de integración. Todo esto supone definir un modelo que, según el tipo y cuantía de stock de capital social relacional (González y Morales, 2006; Morell, 2005).

\section{Marco teórico}

\section{Integración social y Capital social: Los límites de la condición migrante y la emergencia del sujeto migrante mesosocial}

No cabe duda que plantear temas como la integración social en el ámbito migrante genera, de por sí, una dificultad inicial importante para cualquier proyecto de investigación. Y esto se debe al creciente debate que existe sobre la utilidad, pertenencia y validez de dicho concepto o constructo en el ámbito migrante (Moncusí y Rodrigo, 2013). No obstante esto, y considerando lo propuesto por Cachón (2008), la utilidad de este concepto estará dada en la medida que se aclaren las dimensiones que considera el constructo conceptual. En esta línea, y considerando una apuesta similar a la presentada por Moncusí y Rodrigo (2013), nos planteamos tomar parte en la discusión a partir del desarrollo y presentación de una línea conceptual y teórica que permita y facilite el estudio de la integración de los colectivos migrantes como tales en la sociedad de acogida en un nivel mesosocial.

Así, planteamos como supuesto de base que la integración social requiere, en un primer momento, reconocer la particularidad que define al migrante como tal, esto es, lo que ha sido definido como condición (in)migrante (Thayer, 2013; Penninx y Martiniello, 2006), más específicamente, un conjunto de rasgos propios que comparten todos los migrantes asentados en una sociedad distinta a la de origen, y que se pueden resumir en tres dimensiones, a la vez que límites: a) la lógica estadonación que define la diferencia y jerarquización entre nacionales y extranjeros (límite político); b) la dicotomía entre la cultura de origen y la de acogida (límite cultural); y c) la distribución diferencial de los recursos materiales entre nativos e migrantes (límite económico-laboral). Son estos límites los que, en definitiva, terminan por recrear ciertas relaciones particulares (por ejemplo: negación o rechazo cultural, explotación o precarización laboral y exclusión política-electoral) que determinan una distribución de recursos 
desigual entre nativos e migrantes, y con ello, una diversidad de posiciones, generalmente desventajosas para los sujetos migrantes (Lin, 2002). Y es en el proceso de superación de dichos límites cuando los migrantes alcanzan mayores niveles de integración social en la sociedad de acogida (Jensen y Valdebenito, 2010; Thayer, 2013).

En ello, las asociaciones emergen como un agente fundamental en el proceso de integración y separación de los límites de la condición migrante: facilitan la reproducción de las condiciones culturales originales y particulares de los migrantes (Morell, 2005); la orientación hacia el entorno del grupo como una forma de visibilización, sensibilización y posicionamiento del mismo en la sociedad de acogida (Aparicio y Tornos, 2010). También entregan información y oportunidades de trabajo para los desempleados o para los que acaban de llegar (Aparicio y Tornos 2010, Portes y Böröcz 1998). Finalmente, en lo político, las asociaciones son una de las principales alternativas extra electoral en los Estados donde no se reconoce (o se limita de forma considerable) el derecho a sufragio de los migrantes o ciertos colectivos (González y Morales, 2006). Sin embargo, la relevancia del asociacionismo migrante va más allá de su mera funcionalidad, ya que, y como segunda base de su emergencia, este asociacionismo es la expresión de un ethos migrante, es el propio sujeto migrante en un nivel colectivo, es una expresión colectiva y representante de una identidad y cuerpo simbólico propio de los migrantes frente a un "otro" nativo. Y es esto, el ethos migrante, lo que permite entender por qué estos sujetos, más allá de un cálculo racional de costo beneficio, participan y construyen estos sujetos colectivos; y es que además de en tanto entregar recursos, también construyen y facilitan un reconocimiento de su particularidad migrante (del Olmo 2003; Solé y Perella, 2005; Jensen y Valdebenito, 2010). En consecuencia, las asociaciones permiten al colectivo y/o grupo migrante participar en la sociedad de acogida y conseguir sus intereses bajo una relación dinámica y circular entre integración ciudadanía-identidad (Solé y Perella, 2005), o tal como lo explica del Olmo 2003:52:

“Las asociaciones de inmigrantes se convierten en actores sociales, cuyas acciones están orientadas a la formación de una identidad colectiva que permita el reconocimiento de dichos actores sociales. Las características que definen las fronteras de su identidad deben afectar a las relaciones sociales del inmigrante en su nuevo contexto de inmigración, al tiempo que deben permitir una estructura organizativa que se constituya en representante social del colectivo inmigrante reconocido por la sociedad de acogida”

Por tanto, la emergencia del asociacionismo migrante, definido como la movilización propia y exclusiva de los sujetos migrados desde su particularidad (Penninx y Martiniello 2006), constituye un indicador de su integración dada su relación representativa con la sociedad de acogida (Jensen y Valdebenito, 2010; Torres y Garcés, 2013). 


\section{Redes interorganizativas y cívicas del Asociacionismo migrante: de los tipos de relaciones a los tipos de integración social}

La integración social desde una perspectiva relacional encuentra sustento en la propuesta de Berry et al. (2006) cuando se define esta integración como un estadio, entre otros, que se construye según dos principios básicos: la orientación a interactuar entre los colectivos migrantes y nativos, y la consideración de las particularidades culturales, étnicas e identitarias de cada grupo, es decir, "el proceso a través del cual el migrante se convierte en una parte aceptada de la sociedad" (Penninx y Martiniello, 2006). En este contexto, la relevancia de esta perspectiva no está sólo en la consideración relacional de integración, sino también la consideración de un tipo de relación específica, nos referimos a la simetría y autonomía relacional que explicita el respeto/consideración a las particularidades de la otra contraparte, es decir, la emergencia de relaciones horizontales entre los interactuantes, en este caso, entre migrantes y nativos (Sarriera, 2003). Por tanto, el tipo de relaciones que desarrollen las asociaciones con los actores de su entorno es un indicador del capital social reticular desarrollado y, sobretodo, de la base que pueda sustentar su proceso de integración. De no hacer esta diferencia, o lo que es igual, cuantificar y considerar todo tipo de relación, supondría contabilizar, en un primer momento, relaciones asimétricas y verticales como equivalentes a las horizontales, es decir, de otro modo, una relación simétrica, bajo lógicas cooperativas entre distintos actores, cobraría igual significado que las relaciones de tipo asimétricas tales como las que se desarrollan bajo lógicas de asistencialismo, clientelismo, dominación, etc. (Lozares et al., 2011; Cinalli, 2007; Putnam, 2001; Toral, 2009).

En definitiva, si consideramos que el stock de capital social está en relación con los niveles de integración social (Lozares et al., 2011), ya sea desde lo relacional (Coleman, 1990); o también, como señala Putnam (2001), desde lo cívico-cultural en la emergencia del asociacionismo, confianza y formación de redes cívicas; es porque en ambos casos la especificación de la naturaleza de las relaciones es equivalente, esto es, de simetría y horizontalidad (Putnam, 1995; 1993, Ostrom et al., 2003). Por el contrario, las redes o participación en asociaciones fundadas en lógicas de poder o dominación dan lugar a algo distinto, y acaso opuesto, a lo que hemos definido como capital social, tal y como lo afirma el propio Putnam (2011[1993] p.248):

"Una red vertical, por muy densa e importante que sea para sus participantes, no puede mantener la confianza y la cooperación”.

De igual forma, y para el capital social cívico-cultural o redes de compromiso cívico, Millán y Gordon 2004: 734 plantean explícitamente que:

"[La horizontalidad relacional] presupone dos cosas: equidad en la disposición a equilibrar el intercambio y equidad en el trato [entre los interactuantes]", y que se diferencia, en términos de las relacionales, tal como lo ejemplifica Putnam (2011[1993]: 248) cuan- 
do describe que "Las relaciones clientelares, por ejemplo, implican intercambio interpersonal y obligaciones recíprocas, pero el intercambio es vertical y las obligaciones asimétricas [...] una amistad asimétrica; [incluso] las relaciones verticales [caracterizadas como sumisión] parecen socavar la organización de grupos horizontales, y la solidaridad de clientes y patrones por igual, pero especialmente la de los clientes [ya que son] relaciones caracterizadas más por la dependencia que por la reciprocidad”.

Dicho todo esto, las relaciones con el resto de actores del entorno, particularmente los nativos (Estado, sector privado y sociedad civil nativa), variará desde las relaciones de dominación o verticales (clientelismo, asistencialismo, etc.) a las más horizontales (colaboración, cooperación, etc.). Una diferenciación que bien plantea Durston (2000) cuando analiza la formación del capital social en grupos marginados en función de los distintos tipos de relaciones que se tejen entre la sociedad civil y el Estado, esto es, desde el clientelismo hasta las relaciones sinérgicas (colaborativas) o formas de coproducción entre el Estado y la sociedad civil en planos de igualdad y simetría, algo que podría denominarse como la "horizontalidad relacional de las posiciones asimétricas", o como lo define el propio Durston (2000:33), al citar a Woolcock (1998), un eslabonamiento (linkage) sinérgico (cooperativo).

Además, es necesario considerar los tipos de capital social en función de la mayor o menor similitud que existe entre los sujetos interactuantes, máxime si esta consideración la hacemos en la caracterización de relaciones entre grupos sociales distintos, tal como es el caso que se da entre los migrantes y nativos. Así, la diferenciación del capital nace de la distinción más elemental entre lo que se ha denominado como bridging y bonding capital. El primero (o capital social puente) hace referencia a las relaciones heterófilas, estos es, entre conocidos, amigos distantes y participación en asociaciones u organizaciones civiles; por el contrario, el bonding capital (o capital social de unión) se define a partir de relaciones entre miembros homogéneos, es decir, familiares y amigos muy cercanos y/o sujetos pertenecientes a un mismo grupo étnico (Putnam 2001) o también denominadas como relaciones homófilas; idea que Woolcock (1998) resume en dos formas fundamentales del capital social: embeddedness y autonomy. La primera considera relaciones al interior de los grupos; mientras que la otra, la autonomía, hacia el exterior del grupo de referencia. En consecuencia, y tal como lo plantea Aldridge et al. (2002), es posible considerar que el bonding capital actúa como un "pegamento social” al mantener y recrear la cohesión al interior de los grupos; por otro lado, el bridging capital, actuaría como un "aceite social”, ya que permite las relaciones e interacciones entre los sujetos de diferentes grupos.

En consecuencia, la integración social migrante a nivel meso social estará dado por el stock de relaciones interorganizativas horizontales o verticales para con iguales o distintos, y que se resume en la Tabla $\mathrm{N}^{\circ} 1$. 


\section{Tabla N ${ }^{0} 1$ : Modelo clasificatorio de estados de integración mesosocial en función del tipo de relación y contraparte.}

\begin{tabular}{|c|c|c|c|}
\hline \multirow{2}{*}{ Tipo de contraparte relaciones } & \multicolumn{2}{|c|}{ Prevalencia relacional } & \multirow{2}{*}{ Ausencia de relaciones } \\
\cline { 2 - 3 } & $\begin{array}{c}\text { Predominio de } \\
\text { Horizontalidad }\end{array}$ & $\begin{array}{c}\text { Predominio de } \\
\text { Verticalidad }\end{array}$ & A) Autonomia Aislada \\
\hline $\begin{array}{c}\text { Relaciones entre iguales } \\
\text { (Homofilia) }\end{array}$ & E) Cohesión Interna & $\begin{array}{c}\text { C) Dependencia } \\
\text { Intragrupo }\end{array}$ & B) Dependencia Aislada \\
\cline { 2 - 3 } $\begin{array}{c}\text { Relaciones entre distintos } \\
\text { (Heterofilia) }\end{array}$ & F) Autonomía Integrada & $\begin{array}{c}\text { D) Dependencia } \\
\text { Integrada }\end{array}$ & (n) \\
\hline
\end{tabular}

Fuente: elaboración propia a partir de Berry (2006); Lozares (2003 y 2011); Vranken (2001); Jenson (1998); Cinalli (2007).

La frecuencia diferencial de relaciones y tipos de contrapartes da lugar a un continuo de estadios que van desde la autonomía integrada, dado un stock de relaciones "sinérgicas heterófilas", o cohesivas si son "homófilas", pasando por las dependientes, hasta llegar a los estadios de “dependencia y aislamiento”, donde las escasas relaciones fundamentan la subordinación frente a un otro dominante exclusivo (Vranken, 2001).

En esta línea, las relaciones verticales, tal como aquí las hemos definido, responden a un tipo particular de capital social que, si bien teóricamente ha sido escasamente atendido y desarrollado es posible evidenciar y conceptualizar como capital social negativo. Este es un capital que va más a allá de la concepción tradicional entregada por Portes (1998), en tanto meras externalidades negativas del capital social (positivo) (Patulny y Svendsen, 2007). Más bien, este tipo de capital social se construye por la prevalencia de relaciones conflictivas o en la ausencias relacionales de cooperación (Uphoff, 2003), o lo que es igual, un tipo de capital que se despliega bajo relaciones de dominación y control de unos sujetos sobre otros para el beneficio de los dominadores, es decir, relaciones "caracterizadas por la desigual distribución del poder entre los interactuantes” (Jones 2005: 306). En definitiva, la emergencia de estos tipos de capital social son los polos o extremos en un continuo de niveles de integración social de los sujetos migrantes mesosociales.

\section{Métodos y datos}

\section{Metodología del estudio principal}

Los datos se extraen de una encuesta aplicada durante el año 2012, y actualizada en los años2013-14, a una muestra representativa de asociaciones de migrantes, de todo el territorio español (universo de 927 asociaciones) y cuyo tamaño muestral resultó de 225 asociaciones de todos los orígenes exceptuando a las asiáticas. 
La muestra se estratificó según tres variables fundamentales: a) la distribución de la población migrante según su origen en todas las Comunidades Autónomas (CCAA) (Morales et. al., 2008); b) el nivel de concentración de dichos colectivos en las diferentes regiones de España (Fullaondo, 2007) y c) la densidad asociativa de los colectivos migrantes en cada región autónoma (Veredas, 2003).

Lo anterior se logró considerando los datos de distribución de población migrante más reciente al momento de realizar esta investigación (INE, 2011); el listado oficial de asociaciones de migrantes de los registros autonómicos y nacionales y, adicionalmente, el cálculo del denominado "coeficiente de especialización de absorción” de migrantes propuesto por Fullaondo (2007) para cada Comunidad Autónoma. Y que se resume en el siguiente cuadro ${ }^{2}$.

Tabla N²: Afijación muestra

\begin{tabular}{|l|c|}
\hline \multicolumn{1}{|c|}{ Estrato } & $\begin{array}{c}\text { Afijación } \\
\text { Teórica (Muestra Real) }\end{array}$ \\
\hline-1 . Sur-oeste & $45(37)$ \\
\hline-2 . Centro-Este & $50(44)$ \\
\hline-3. Nor-oeste & $38(37)$ \\
\hline
\end{tabular}

Fuente: Elaboración propia / Las cifras entre paréntesis indican la muestra real analizada.

\section{Instrumento}

El cuestionario aplicado a las asociaciones se realizó por medio de encuestas online utilizando el sistema CAWI, para esto se confeccionó un listado con todos los correos electrónicos de las asociaciones; además, y en caso de no contar con correo electrónico o si este era erróneo, se contactó por teléfono a directivos/as de la asociación a fin de corregir/obtener el correo electrónico válido y, de esa forma, proceder al envío de la encuesta.

El instrumento evalúa dos aspectos fundamentales:

- Tipo de contraparte: Si se trata de una entidad de tipo estatal, privada, de la sociedad civil nativa o inmigrante (otras asociaciones de migrantes).

- Tipos de relaciones: según el diferencial de poder que se crea en la relación diádica entre las asociaciones y los actores a partir de la capacidad de control de cada una de las partes en las actividades y acciones que conjuntamente desarrollaron.

A partir de estas preguntas se procedió, por un lado, a definir la cuantía de relaciones según el tipo de contraparte y, seguido de ello, cuan- 
tificar el grado de influencia que tenía tanto la asociación como la contraparte identificada respecto de las actividades que se definieron en la relación, es decir, los diferenciales decisionales para cada relación descrita; y que en conjunto permitió cuantificar los patrones relacionales (horizontales v/s verticales) que desarrollaban tanto las asociaciones como los actores nativos.

\section{Análisis de los datos}

\section{Distribución de las relaciones interorganizativas}

Para evaluar el modelo propuesto en términos teóricos se hizo necesario identificar los tipos de contrapartes con los que las asociaciones afirman relacionarse. Para esto, consultamos por estas contrapartes según seis grupos finales. Los resultados se exponen de forma descriptiva en la siguiente Tabla $\mathrm{N}^{\circ} 3$.

Tabla N ${ }^{\circ} 3$ : Relaciones interorganizativas entre grupos de asociaciones (por origen) y tipos de contrapartes ${ }^{3}$

\begin{tabular}{|c|c|c|c|c|c|c|c|c|}
\hline & & $\begin{array}{c}\text { (a) } \\
\text { Autoridade } \\
\mathrm{s} \\
\text { Españolas }\end{array}$ & $\begin{array}{c}\text { (b) } \\
\text { Asociacione } \\
\mathrm{s} \\
\text { migrantes }\end{array}$ & $\begin{array}{c}\text { (b) } \\
\text { Autoridades } \\
\text { o Entidades } \\
\text { migrantes } \\
\text { en/de origen }\end{array}$ & $\begin{array}{c}\text { (c) } \\
\text { Fundaciones } \\
\text { y Ongs } \\
\text { Nativas }\end{array}$ & $\begin{array}{c}(c) \\
\text { Organizacion } \\
\text { es Socio } \\
\text { comunitarias } \\
\text { Nativas }\end{array}$ & $\begin{array}{c}\text { (d) } \\
\text { Empresa } \\
\text { s } \\
\text { Nativas }\end{array}$ & Total \\
\hline \multirow{2}{*}{ África } & $\mathrm{N}$ & 56 & 74 & 5 & 72 & 40 & 14 & \multirow{2}{*}{$\begin{array}{r}45 \\
(279) \\
\end{array}$} \\
\hline & $\%$ & 124,4 & 164,4 & 11,1 & 162,2 & 88,9 & 31,1 & \\
\hline \multirow{2}{*}{$\begin{array}{l}\text { E Este } \\
\text { ExURSS }\end{array}$} & $\mathrm{N}$ & 49 & 58 & 8 & 54 & 28 & 19 & \multirow{2}{*}{$\begin{array}{r}33 \\
(225) \\
\end{array}$} \\
\hline & $\%$ & 148,5 & 175,8 & 24,2 & 163,6 & 84,8 & 57,6 & \\
\hline \multirow{2}{*}{$\begin{array}{l}\text { Pluri } \\
\text { nacional }\end{array}$} & $\mathrm{N}$ & 80 & 83 & 5 & 119 & 43 & 37 & \multirow{2}{*}{$\begin{array}{r}48 \\
(376)\end{array}$} \\
\hline & $\%$ & 166,7 & 172,9 & 10,4 & 247,9 & 89,6 & 71,1 & \\
\hline \multirow{2}{*}{ Latino } & $\mathrm{N}$ & 108 & 193 & 9 & 134 & 73 & 67 & \multirow{2}{*}{$\begin{array}{r}84 \\
(603) \\
\end{array}$} \\
\hline & $\%$ & 128,6 & 229,8 & 10,7 & 160,7 & 86,9 & 79,8 & \\
\hline TOTAL & & 293 & 408 & 27 & 381 & 184 & 137 & $\begin{array}{r}210 \\
(1430) \\
\end{array}$ \\
\hline
\end{tabular}

Fuente: elaboración propia

Los datos consolidados revelan algunos elementos centrales en la conformación de la red de relaciones interorganizativas. Por ejemplo, la preeminencia de relaciones con entidades de tipo inmigrantes como fenómeno transversal a los distintos tipos de asociaciones y, sobre todo, para las asociaciones latinas, ya que cada una de ellas declara, al menos, dos relaciones con otras asociaciones de inmigrantes. En total, este tipo de contrapartes concentra 408 relaciones, y significa un $28,5 \%$ del total de relaciones que se declaran.

Un segundo grupo de contrapartes relevante son las denominadas "Fundaciones y ONGs nativas” con 381 relaciones, esto es, un 26,6\% del total, y 
donde destaca el alto porcentaje de las asociaciones plurinacionales, esto es, cada asociación plurinacional desarrolla más de 2,5 relaciones con este tipo de contrapartes. Una tendencia similar se observa en el caso de las Autoridades Españolas o nativas que concentran, por sí solas, casi el 30\% de las relaciones totales, y donde las asociaciones plurinacionales alcanzan, nuevamente, una importante frecuencia relacional, al igual que las asociaciones latinas que particularizan su stock relacional bajo coordenadas más de tipo endogámica.

Del resto de contrapartes cabe resaltar las particularidades de las contrapartes sociocomunitarias que se alazán como el cuarto actor más relevante con un 80\% de relaciones en la muestra, que aunque extensa, se observa cierta homogeneidad relacional con todos los tipos de asociaciones. Respecto de las contrapartes de tipo “comercial”, si bien alcanzan el último lugar de relevancia, es notorio que las asociaciones africanas son las que menos relaciones definen con este tipo de entidades, más de un 20\% de diferencia respecto de las asociaciones de Europa del Este, las siguientes en desarrollar menos relaciones con estas entidades del sector privado.

\section{Centralidad de grado ponderado}

Una de las primeras medidas consideradas en el análisis y definición del modelo se evidencia a partir de la frecuencia de relaciones con los actores del entorno o centralidad de grado ponderado que puntúa cada una de las asociaciones entrevistadas, tal y como se describe en la TablaNº 4 .

Tabla Nº: Grado ponderado por grupos de asociaciones según origen.

\begin{tabular}{|c|c|c|c|c|c|c|}
\hline \multirow{2}{*}{\multicolumn{2}{|c|}{$\begin{array}{l}\text { Grupo de } \\
\text { nacionalidades }\end{array}$}} & \multicolumn{5}{|c|}{ Niveles de grado ponderado (relaciones) según cuartiles } \\
\hline & & \multirow{2}{*}{$\begin{array}{l}\text { Ninguna o Casi } \\
\text { Ninguna (de } 0 \\
\text { a 2,4) } \\
\end{array}$} & \multirow{2}{*}{$\begin{array}{c}\text { Algunas } \\
\begin{array}{c}\text { (de } 2,41 \mathrm{a} \\
4,40)\end{array} \\
16\end{array}$} & \multirow{2}{*}{$\begin{array}{r}\text { Bastantes } \\
(\text { de } 4,41 \mathrm{a} \\
7,20) \\
16\end{array}$} & \multirow{2}{*}{$\begin{array}{l}\text { Muchas } \\
\text { (más de } \\
7,21) \\
\end{array}$} & \multirow{2}{*}{$\begin{array}{r}\text { Total } \\
51 \\
\end{array}$} \\
\hline \multirow{3}{*}{ África } & $\mathrm{N}$ & & & & & \\
\hline & $\%$ & $27,5 \%$ & $31,4 \%$ & $31,4 \%$ & $9,8 \%$ & $100,0 \%$ \\
\hline & RTC & 3 & 1,1 & 9 & $-2,4$ & \\
\hline \multirow{3}{*}{$\begin{array}{l}\text { Europa del } \\
\text { Estey } \\
\text { ExURSS }\end{array}$} & $\mathrm{N}$ & 11 & 8 & 9 & 9 & 37 \\
\hline & $\%$ & $29,7 \%$ & $21,6 \%$ & $24,3 \%$ & $24,3 \%$ & $100,0 \%$ \\
\hline & RTC & ,6 &,- 6 &,- 4 &, 3 & \\
\hline \multirow{3}{*}{ Plurinacional } & $\mathrm{N}$ & 13 & 10 & 10 & 17 & 50 \\
\hline & $\%$ & $26,0 \%$ & $20,0 \%$ & $20,0 \%$ & $34,0 \%$ & $100,0 \%$ \\
\hline & RTC &, 0 & $-1,0$ & $-1,2$ & 2,3 & \\
\hline \multirow{3}{*}{ Latino } & $\mathrm{N}$ & 20 & 23 & 25 & 19 & 87 \\
\hline & $\%$ & $23,0 \%$ & $26,4 \%$ & $28,7 \%$ & $21,8 \%$ & $100,0 \%$ \\
\hline & RTC &,- 8 &, 3 &, 6 &,- 1 & \\
\hline \multirow{2}{*}{ Total } & $\mathrm{N}$ & 58 & 57 & 60 & 50 & 225 \\
\hline & $\%$ & $25,8 \%$ & $25,3 \%$ & $26,7 \%$ & $22,2 \%$ & $100,0 \%$ \\
\hline
\end{tabular}

Fuente: elaboración propia / RTC $=$ Residuos Tipificados Corregidos. Nota: La proporción de cada cuartil no es del $25 \%$ exacto debido a que son considerables los casos que alcanzan puntuaciones similares a los valores de corte para definir los cuartiles. 
Bajo esta clasificación podemos evidenciar que las asociaciones se distribuyen de forma particular, aunque no significativa, según su origen o nacionalidad. Así, las asociaciones africanas se concentran en las categorías con menos relaciones y, además, son las que menos recrean "muchas relaciones", un perfil similar se observa para las asociaciones de Europa del Este que, y a diferencia de las africanas, si posicionan un número considerable de asociaciones en la categoría de "muchas relaciones". Respecto de las asociaciones latinas se observa una distribución bastante homogénea en todas las categorías, algo que no se observa para las plurinacionales que son, con diferencia, las que, en mayor proporción, reflejan una cantidad considerable de relaciones.

\section{Tendencia a la Exogamia - Endogamia de las relaciones interorganizativas}

En este apartado pretendemos dar cuenta de una segunda dimensión propia de la integración de las asociaciones migrantes: la tendencia que detentan a desarrollar relaciones más orientadas a la endogamia o, por el contrario, a la exogamia. En lo específico, consideramos la centralidad de grado que cada asociación distribuye entre actores migrantes (endogamia) y/o entre los nativos españoles (exogamia).

En esta línea, y según los datos de la Tabla $\mathrm{N}^{\circ} 5$, podemos ver que la distribución de estas tendencias relacionales tiene alguna relación con el origen de las asociaciones. Así, las asociaciones denominadas plurinacionales son las que concentran un mayor número de casos en los que se privilegia las relaciones exogámicas (80\%), patrón que también se repite, en el resto de asociaciones aunque con ciertas particularidades. Por ejemplo, las asociaciones de Europa del Este se posicionan en menor medida que el resto de asociaciones en estadios de endogamia y mucho más, en términos comparados, en posiciones más bien neutras o equilibradas, y es que casi un $20 \%$ de estas asociaciones desarrollan un equilibrio entre las relaciones que establecen con entidades migrantes y las que establecen con actores nativos; algo que es opuesto a la tendencia de las asociaciones Latinoamericanas ya que describen una cierta concentración relacional bajo lógicas endogámicas, todo, si hacemos la comparación con las distribuciones del resto de colectivos. Esto no es de extrañar si pensamos que estas mismas asociaciones latinas actúan también como contrapartes transversales y relevantes a un gran número de la muestra. Por último, las asociaciones africanas se asemejan bastante a la distribución de la muestra total, lo que implica que gran parte de sus relaciones las orientan hacia los actores nativos. 
Polis, Revista Latinoamericana, Volumen 14, No 42, 2015

Tabla Nº5: Distribución de relaciones interorganizativas según tendencia a la endogamia-exogamia y origen de las asociaciones.

\begin{tabular}{|c|c|c|c|c|c|}
\hline & \multicolumn{4}{|c|}{ Tendencia de las relaciones } \\
\hline & & Endogamia & Neutro & Exogamia & Total \\
\hline \multirow{3}{*}{ África } & $\mathrm{N}$ & 5 & 7 & 39 & 51 \\
\hline & $\%$ & $9,8 \%$ & $13,7 \%$ & $76,5 \%$ & $100,0 \%$ \\
\hline & RTC &,- 5 & 3 & 2 & \\
\hline \multirow{3}{*}{ Europa del Este y ExURSS } & $\mathrm{N}$ & 1 & 7 & 29 & 37 \\
\hline & $\%$ & $2,7 \%$ & $18,9 \%$ & $78,4 \%$ & $100,0 \%$ \\
\hline & RTC & $-1,9$ & 1,3 &, 4 & \\
\hline \multirow{3}{*}{ Plurinacional } & $\mathrm{N}$ & 4 & 6 & 40 & 50 \\
\hline & $\%$ & $8,0 \%$ & $12,0 \%$ & $80,0 \%$ & $100,0 \%$ \\
\hline & RTC & $-1,0$ &,- 1 & 8 & \\
\hline \multirow{3}{*}{ Latino } & $\mathrm{N}$ & 17 & 8 & 62 & 87 \\
\hline & $\%$ & $19,5 \%$ & $9,2 \%$ & $71,3 \%$ & $100,0 \%$ \\
\hline & RTC & 2,8 & $-1,2$ & $-1,2$ & \\
\hline \multirow{2}{*}{ Total } & $\mathrm{N}$ & 27 & 28 & 170 & 225 \\
\hline & $\%$ & $12,0 \%$ & $12,4 \%$ & $75,6 \%$ & $100,0 \%$ \\
\hline
\end{tabular}

Fuente: elaboración propia $/$ RTC $=$ Residuos Tipificados Corregidos

\section{Niveles de simetría y asimetría relacional en las relaciones interorganizativas}

Una tercera dimensión en la construcción del modelo se relaciona con el grado de simetría relacional que existe entre los sujetos interactuantes. En este caso consideramos como simétricas a todas aquellas relaciones en donde se explicita, por parte de las asociaciones, que durante la relación ambas partes (la asociación y su contraparte) tenían igual nivel de influencia/poder a la hora de tomar decisiones en los aspectos concernientes a la actividad que los convocaba; por el contrario, la asimetría se define como un diferencial decisional a favor de alguna de las partes interactuantes.

En este contexto, evaluamos estas distribuciones en función del origen de las asociaciones, tal y como se muestra en la Tabla $\mathrm{N}^{\circ} 6$, algo que nos permite constatar ciertas regularidades, aunque no significativas, entre los distintos colectivos. Particularmente, que las asociaciones de los colectivos de Europa del Este y Ex URSS y plurinacionales tienden, mayoritariamente y en términos comparativos, a concentrarse en posiciones más bien neutras, alcanzando más de un $20 \%$ en cada una de estas posiciones y con un $10 \%$ más sobre el resto de colectivos para esta misma categoría. Esta tendencia a neutralizar sus stock relacionales con el entorno son diferenciadas para ambos tipos: plurinacionales y de Europa del Este. Así, por un lado las asociaciones de Europa del Este lo hace en desmedro de sus posiciones ya que es el tipo de asociación que alcanza una menor 
proporción en este tipo de estadios; por el contrario, las asociaciones plurinacionales lo hace en desmedro de las posiciones asimétricas, alcanzando cerca de siete puntos porcentuales menos que el resto de colectivos. Por otro lado, si bien las asociaciones africanas alcanzan proporciones similares en estadios asimétricos que los de Europa del Este, es propio de estas asociaciones tener una importante presencia en estadios simétricos. Por último, si bien el colectivo latino se asemeja, en alguna medida, a la distribución de las asociaciones africanas, define con mayor claridad su tendencia hacia la simetría, o al menos, una leve tendencia menor a concentrarse en estadios asimétricos. Tabla Nº: Distribución de relaciones interorganizativas según tenden-
cia a la simetría-asimetría y origen de las asociaciones.

\begin{tabular}{|c|c|c|c|c|c|}
\hline & & \multicolumn{4}{|c|}{ Tendencia de las relaciones } \\
\hline & & Asimetría & Neutro & Simetría & Total \\
\hline \multirow{3}{*}{ África } & $\mathrm{N}$ & 22 & 4 & 25 & 51 \\
\hline & $\%$ & $43,1 \%$ & $7,8 \%$ & $49,0 \%$ & $100,0 \%$ \\
\hline & RTC & 4 & $-1,4$ & .5 & \\
\hline \multirow{3}{*}{$\begin{array}{l}\text { Europa del Este y } \\
\text { Ex URSS }\end{array}$} & $\mathrm{N}$ & 16 & 8 & 13 & 37 \\
\hline & $\%$ & $43,2 \%$ & $21,6 \%$ & $35,1 \%$ & $100,0 \%$ \\
\hline & RTC & 4 & 1,5 & $-1,4$ & \\
\hline \multirow{3}{*}{ Plurinacional } & $\mathrm{N}$ & 18 & 10 & 22 & 50 \\
\hline & $\%$ & $36,0 \%$ & $20,0 \%$ & $44,0 \%$ & $100,0 \%$ \\
\hline & RTC &,- 7 & 1,4 &,- 3 & \\
\hline \multirow{3}{*}{ Latino } & $\mathrm{N}$ & 35 & 9 & 43 & 87 \\
\hline & $\%$ & $40,2 \%$ & $10,3 \%$ & $49,4 \%$ & $100,0 \%$ \\
\hline & RTC &,- 1 & $-1,2$ & ,9 & \\
\hline \multirow{2}{*}{ Total } & $\mathrm{N}$ & 91 & 31 & 103 & 225 \\
\hline & $\%$ & $40,4 \%$ & $13,8 \%$ & $45,8 \%$ & $100,0 \%$ \\
\hline
\end{tabular}

Fuente: elaboración propia $/$ RTC $=$ Residuos Tipificados Corregidos

Pero más allá de esta particularidad, nuestro objetivo es ver cómo estas distribuciones dan cuenta, en su conjunto, de los distintos estadios de integración que hemos definido con anterioridad. Y esto, en términos resumidos considera los tres elemento ante señalados: a) una primera base en función de la cuantía de relaciones interorganizativas, más particularmente en la ausencia de relaciones que da lugar a estadios de "autonomía aislada”, y "dependencia aislada” para el caso en que las pocas relaciones se definan con entidades nativas. En una segunda base, las restantes asociaciones se clasifican b) en función de si las relaciones se desarrollan con entidades migrantes (endogamia/homofilia) o nativas (exogamia/heterofilia) y si en éstas c) priman la simetría o asimetría y, según sea la combinatoria de estas categorías, se definen los estadios correspondientes, tal y como se 
muestra en la Tabla $\mathrm{N}^{0} 7$ donde las asociaciones se clasifican en uno de los siguientes estadios.

\section{Tabla N²: Cuantificación de los estadios de integración según la distribución de las relaciones interorganizativas en función del número de relaciones, niveles de endogamia y niveles de simetría ${ }^{4}$.}

\begin{tabular}{|c|c|c|c|}
\hline \multirow{2}{*}{ Tipo de contraparte } & \multicolumn{2}{|c|}{ Prevalencia significativa de las relaciones } & \multirow{2}{*}{$\begin{array}{c}\text { Ausencia significativa } \\
\text { de relaciones }\end{array}$} \\
\hline & $\begin{array}{l}\text { Predominio de } \\
\text { Horizontalidad }\end{array}$ & $\begin{array}{c}\text { Predominio de } \\
\text { Verticalidad }\end{array}$ & \\
\hline $\begin{array}{c}\text { Relaciones "Intra"; } 0 \\
\text { entre iguales } \\
\text { ENDOGAMIA }\end{array}$ & $\begin{array}{c}\text { E) Cohesión Interna } \\
\text { (Bonding social capital) } \\
\text { Frecuencia: } 15 \\
\text { Porcentaje: } 6,7 \%\end{array}$ & $\begin{array}{l}\text { C) Dependencia } \\
\text { intragrupo } \\
\text { Frecuencia: } 7 \\
\text { Porcentaje: } 3,1 \%\end{array}$ & $\begin{array}{c}\text { A) Autonomía Aislada } \\
\text { Frecuencia: } 4 \\
\text { Porcentaje: } 1,8 \%\end{array}$ \\
\hline $\begin{array}{c}\text { EXOGAMIA } \\
\text { Relaciones "Entre } \\
\text { distintos y diferentes" }\end{array}$ & $\begin{array}{c}\text { F)Autonomía Integrada } \\
\text { (Linking y } \\
\text { Bridgingsocial capital) } \\
\text { Frecuencia: } 68 \\
\text { Porcentaje: } 30,2 \%\end{array}$ & $\begin{array}{c}\begin{array}{c}\text { D) Dependencia } \\
\text { Integrada }\end{array} \\
\text { Frecuencia: } 66 \\
\text { Porcentaje: }: 29,3 \%\end{array}$ & 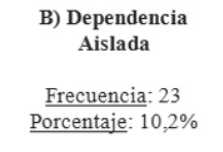 \\
\hline
\end{tabular}

Fuente: elaboración propia

La consolidación de las diferentes variables nos permite analizar la distribución de las asociaciones en los distintos estadios de integración. Y donde lo evidente y relevante es la clara tendencia que muestran (Tabla $\mathrm{N}^{\circ} 7$ ), en general, las asociaciones de migrantes para posicionarse en ambos estadios definidos como exogámicos: tanto el dependiente como el independiente o autónomo. Desde la perspectiva de simetría (columnas) observamos que las relaciones horizontales, tanto endogámicas como exogámicas, tienen una cierta preeminencia $(36,9 \%)$ sobre las verticales $(32,4)$. En cualquier caso, un hecho interesante que se desprende, bajo esta definición relacional, es que no parece haber una clara y evidente situación de dependencia o dominio por parte de las entidades nativas sobre las asociaciones, más bien existe un cierto margen de maniobra en donde las asociaciones definen buena parte de sus relaciones bajo lógicas simétricas. Una excepción a esto son los casos definidos como "ausencia significativa de relaciones", donde se constata la frecuencia de asociaciones que no desarrollan un número suficientemente relevante de relaciones con el entorno, y que dejan a este grupo de asociaciones, un $25 \%$ de la muestra, con escasas capacidades de insertarse en la red interorganizativa, tanto a nivel endogámico como exogámico.

Además de lo anterior, la distribución de estas relaciones simétricas nos habla de la formación de diferentes tipos de capital social, aunque en distintas proporciones. En función de las relaciones simétricas exogámicas podemos considerar la formación de capital social del tipo bridging y (eventualmente) linking capital (30,2\%), por otro lado, se evidencia un escaso 
stock de bonding capital, cercano al 6,7\% (stock de relaciones simétricas endogámicas). Es de esperar, entonces, que el conjunto de relaciones simétricas se funden sobre acciones relacionales esporádicas y con un alto costo para ser recreadas, aunque también, esto conlleva altas posibilidades de acceder a recursos variados y estratégicos, más aún si llegamos a considerar que parte importante de este stock de relaciones simétricas exogámicas responden a relaciones que fundamentan el linking capital.

Por otro lado, es necesario resaltar que un porcentaje no menor de asociaciones, 29,3\%, desarrolla mayoritariamente sus relaciones con el entorno bajo relaciones exogámicas asimétricas o verticales y, también, un 3,1\% con igual base vertical pero con orientación endogámica, lo que en su conjunto nos habla de que casi 1 de cada 3 asociaciones no se encuentra en posiciones relacionales favorables para su integración ya que sus relaciones son recreadas principalmente bajo lógicas de asimetría, o lo que es igual, de control o poder por una contraparte en el desarrollo de las actividades y acciones compartidas y que, según la distribución de los datos, se podría afirmar que son principalmente actores nativos.

\section{Estadio de integración según origen de la asociación}

Además de lo anterior, nuestro interés también radica en identificar hasta qué punto el origen y/o nacionalidad de las asociaciones juega un papel en el desarrollo de los distintos estadios y tipos de capital social, para ello presentamos la siguiente Tabla $\mathrm{N}^{\circ} 8$ que recoge los cruces correspondientes entre tipo de asociación según origen o nacionalidad y estadio de integración.

\section{Tabla Nº: Estadios de integración de las asociaciones según origen.}

\begin{tabular}{|c|c|c|c|c|c|c|c|c|c|}
\hline & & \multicolumn{8}{|c|}{ Estadios de integración } \\
\hline & & $\begin{array}{c}1 \\
\text { Autonomia } \\
\text { Aislada }\end{array}$ & $\begin{array}{c}2 \\
\text { Dependencia } \\
\text { Asilada }\end{array}$ & $\begin{array}{c}3 \\
\text { Dependencia } \\
\text { intragrupo }\end{array}$ & $\begin{array}{c}4 \\
\text { Dependencia } \\
\text { Integrada }\end{array}$ & $\begin{array}{c}5 \\
\text { Cohesión } \\
\text { interna }\end{array}$ & $\begin{array}{c}6 \\
\text { Autonomia } \\
\text { integrada }\end{array}$ & $\begin{array}{l}\text { Neutro } \\
\text { (sin } \\
\text { clasificar) }\end{array}$ & Total \\
\hline \multirow{3}{*}{ África } & $\mathrm{N}$ & 0 & 8 & 1 & 17 & 4 & 14 & 7 & 51 \\
\hline & $\%$ & 0,0 & 15,7 & 2,0 & 33,3 & 7,8 & 27,5 & 13,7 & \\
\hline & RTC & $-1,1$ & 1,5 & $-0,5$ & 0,7 & 0,4 & $-0,5$ & $-1,0$ & \\
\hline \multirow{3}{*}{$\begin{array}{l}\text { Europa del } \\
\text { Este } \\
\text { y Ex URSS }\end{array}$} & $\mathrm{N}$ & 0 & 2 & 1 & 13 & 0 & 11 & 10 & 37 \\
\hline & $\%$ & 0,0 & 5,4 & 2,7 & 35,1 & 0,0 & 29,7 & 27,0 & \\
\hline & RTC & $-0,9$ & $-1,1$ & $-0,2$ & 0,8 & $-1,8$ & $-0,1$ & 1,4 & \\
\hline \multirow{3}{*}{$\begin{array}{l}\text { Pluri } \\
\text { nacional }\end{array}$} & $\mathrm{N}$ & 0 & 5 & 1 & 12 & 3 & 16 & 13 & 50 \\
\hline & $\%$ & 0,0 & 10,0 & 2,0 & 24,0 & 6,0 & 32,0 & 26,0 & \\
\hline & RTC & $-1,1$ & $-0,1$ & $-0,5$ & $-0,9$ & $-0,2$ & 0,3 & 1,5 & \\
\hline \multirow{3}{*}{ Latino } & $\mathrm{N}$ & 4 & 8 & 4 & 24 & 8 & 27 & 12 & 87 \\
\hline & $\%$ & 4,6 & 9,2 & 4,6 & 27,6 & 9,2 & 31,0 & 13,8 & \\
\hline & RTC & 2,5 & $-0,4$ & 1,0 & $-0,5$ & 1,2 & 0,2 & $-1,5$ & \\
\hline \multirow{2}{*}{ Total } & $\mathrm{N}$ & 4 & 23 & 7 & 66 & 15 & 68 & 42 & 225 \\
\hline & $\%$ & 1,8 & 10,2 & 3,1 & 29,3 & 6,7 & 30,2 & 18,7 & \\
\hline
\end{tabular}

Fuente: elaboración propia $/$ RTC $=$ Residuos Tipificados Corregidos 
Tal como se observa en la Tabla No8 la distribución de las asociaciones, según su origen/nacionalidad, entre los distintos estadios de integración no resulta significativa a nivel estadístico (probablemente por un bajo número de casos respecto al alto número de casillas), sin embargo, sí parecen existir algunos patrones que pueden ser considerados, al menos, como descriptivos.

Así, y bajo la comparación de las distribuciones porcentuales a través del continuo de estadios formados por las columnas de la Tabla $N^{\circ} 8$, donde las categorías más hacia la izquierda nos hablan de estadios menos favorables respecto de los situados más hacia la derecha, ("autonomía integrada”) para propiciar estadios propios de la integración social, al menos, tal y como la hemos definido en esta investigación. Específicamente, vemos que en la comparación de los cuatro primeros estadios, los colectivos latinos y plurinacionales alcanzan puntuaciones más cercanas a estadios de integración respecto de las obtenidas por los colectivos Africanos y de Europa del Este (residuos tipificados corregidos). Esta tendencia resulta aún más evidente cuando la comparación se focaliza en el estadio de "dependencia integrada” (prevalencia de relaciones exogámicas asimétricas) donde los colectivos africanos y europeos del este obtienen diferencias a su favor cercanas al $10 \%$ respecto de las asociaciones plurinacionales y latinas.

En lo que respecta a los estados propios de mayor integración social y de formación del capital social integrador, vemos que estas tendencias entre colectivos parecieran tener alguna correlación. En lo particular, vemos que las asociaciones latinas y plurinacionales son los que alcanzan mayores proporciones de asociaciones en estados de “autonomía integrada” o linking y bridging capital y, para el caso de los latinos, también en las relaciones autónomas de carácter endogámicas o bonding capital. A diferencia de ellos, los colectivos africanos, y sobre todo los de Europa del Este, obtienen puntuaciones más bajas en la "autonomía integrada”, y sobre todo para estos migrantes europeos en lo que concierne a la "cohesión interna"; todo lo cual nos entrega, de alguna forma, un nuevo antecedente de cómo se integran las asociaciones migrantes según su origen y los colectivos que representan, y que se alinea con las conclusiones preliminares obtenidas al momento de hacer el análisis descriptivo de cada una de las variables por separado.

En consecuencia, podríamos afirmar que las asociaciones latinas alcanzan una mayor proporción y participación en los estadios "más integrados” respecto del resto de colectivos; en un segundo lugar lo serían las asociaciones plurinacionales y, finalmente, las asociaciones de origen africano, cuya proporción en estadios de “dependencia aislada” es una de los más considerables. Sin embargo, parecen ser las asociaciones de Europa del Este las que detentan una posición global menos favorable, ya que se distribuyen con menor frecuencia en la "autonomía integrada”, con una alta presencia en la “dependencia integrada” y, además, son el único grupo que tiene nula presencia en estadios de “cohesión interna”. 


\section{Conclusiones}

La definición de la propuesta modélica presentada en este trabajo nos permite evidenciar, como primera conclusión, que la mayoría de las asociaciones acumula y desarrolla un stock importante de relaciones con actores del entorno. Sin embargo, estas relaciones presentan evidentes diferenciales de poder entre las partes interactuantes, y más aún en las relaciones exogámicas que en las endogámicas. Esto último, la simetría endogámica, se evidencia en dos de cada tres relaciones orientadas a actores de naturaleza migrante, una propiedad que no es más que la confirmación de ciertos patrones y atributos propios de las denominadas relaciones homófilas, en tanto recreadas entre iguales (en este caso entre asociaciones y/o entidades migrantes), y que conforman la base relacional propia para el surgimiento de un capital social del tipo bonding capital, y como stock relacional relevante define uno de los estadios de nuestro modelo, el de "cohesión interna", que en sí juega un papel relevante en tanto base para el desarrollo de la sociedad civil inmigrante y al favorecer la cohesión interna de los colectivos y del mundo inmigrante en general, y donde sobresale, como principal exponente, la asociación inmigrante de origen latinoamericano, ya que es el tipo de asociación que más facilita la estructura relacional interna inmigrante. No obstante lo anterior, esta orientación relacional endogámica también se recrea y expresa bajo relaciones cooperativas con rasgos asimétricos, y aunque representa un porcentaje muy escaso, da lugar a un segundo estadio de integración definido como "dependencia intragrupo".

Por otro lado, las relaciones heterófilas y/o exogámicas, que son las más numerosas y bases para la definición de la integración social, dan cuenta de dos estadios de integración diferenciados y con referencia a una "integración independiente” (relaciones exogámicas-simétricas) y que sienta las bases de la integración social a partir de los dos tipos de capital social de tipo exogámico: el bridging y linking capital. El primero, vemos que se explicita a partir de las relaciones que las asociaciones establecen con entidades de la sociedad civil, principalmente ONGs generalistas y locales; y el segundo, el linking, que es más escaso y menos evidente con ciertas autoridades locales y supralocales. En consecuencia, es posible concluir que las asociaciones tienen claras referencias relacionales hacia el entorno, y sobre el cual construyen, principalmente, capitales sociales de tipo bonding con asociaciones latinas y bridging con organizaciones de la sociedad civil.

Por otro lado, el resto de relaciones exogámicas definen los estadios opuestos a los descritos, estos se caracterizan por desarrollarse bajo la asimetría negativa y dan cuenta de lo que definimos como "capital social negativo". Particularmente nos referimos al estadio denominado como "integración dependiente" que es el que congrega el mayor número de asociaciones, específicamente, el 30\% de la muestra consultada mantienen relaciones primordialmente asimétricas con actores nativos. Esto resulta relevante si pensamos que este tipo de capital social, el negativo, actúa como 
base para reproducir y perpetuar estadios de dependencia en algunos casos y/o de subordinación en otros, impidiendo, por un la lado, el despliegue autónomo de las asociaciones y, por otro, la desigual distribución de poder entre los interactuantes, entre ello, también los recursos. Uno de los ejemplos paradigmáticos de perpetuar estos estadios se observa en la limitación programática que enfrentan las asociaciones cuando deben subordinar su actuar frente a las disposiciones de sus patrocinadores, es decir, las asociaciones pueden quedar encauzadas, y a veces obligadas, a realizar ciertas actividades según la conveniencia del proveedor, algo más evidente y relevante para el caso de las asociaciones más políticas y estratégicas, en tanto queda limitada su capacidad reivindicativa y de oposición frente a las autoridades; situación ésta que ha sido conceptualizada y definida en el ámbito del asociacionismo inmigrante como cooptación; esto es, las asociaciones quedan relegadas al ámbito asistencial y maniatadas en el ejercicio reivindicativo (en caso que tal hubiera existido) frente a quien es su mentor y/o principal proveedor de recursos (Toral 2010; Veredas 2003).

Para el resto de estadios de integración propuestos en el modelo se definen principalmente por el escaso o nulo stock de relaciones interorganizativas; y en caso de haberlas, dichas relaciones se definen en función de la asimetría a favor de las contrapartes nativas. Por ejemplo, se da lugar al estadio de "dependencia aislada”, que si bien no es la categoría más relevante, si logra un $10 \%$ de los casos. Por el contrario, el estadio “autonomía aislada” no logra congregar a más del 2\% de la muestra, y agrupa a las asociaciones que desarrollan un bajo stock de relaciones con el "mundo inmigrante". En cualquier caso, la principal característica de estos dos últimos estadios es el hecho que, al menos, una de cada 4 asociaciones desarrolla pocas o ninguna relación con el entorno, lo que supone una situación de claro aislamiento y la imposibilidad (incluso bajo la modalidad dependencia) de que estas asociaciones puedan participar del entramado reticular que se desarrolla en la sociedad de acogida.

Finalmente, y respecto del origen o nacionalidad de las asociaciones, si bien la falta de significatividad estadística no nos permite confirmar que dicho origen sea una variable asociada a la integración social, todo según el modelo de clasificación que hemos diseñado; se obtiene, igualmente, evidencia sobre la persistencia de ciertos patrones asociados al origen de las asociaciones, tal es el caso de las asociaciones Latinas respecto de las de Europa del Este, por citar los casos más extremos, y donde los primeros revelan con claridad y diferencia que, por ejemplo, poseen mayores niveles de relaciones exogámicas simétricas y cumplen un significativo papel cohesionador en el conjunto de las asociaciones migrantes, tanto a nivel grupal como en términos diádicos. En conclusión, es posible aventurar que, en términos generales, existen antecedentes y evidencia suficiente como para considerar que el origen de las asociaciones se relaciona con distintos estadios de integración social. 


\section{Notas}

${ }^{1}$ Artículo desarrollado en el marco del proyecto Fondecyt / postdoctorado $\mathrm{N}^{\circ}$ 3150449, del cual el autor José Manuel Gaete es su investigador responsable. La investigadora Paola Ilabaca está adscrita al Centro CIELO y es académica de la Escuela de Psicología sede Santiago, de la Universidad Santo Tomás.

${ }^{2}$ Si bien no se logró completar la cuota de la afijación teórica propuesta para cada estrato, aun cuando se contactó a la totalidad del marco muestral, la muestra consultada alcanza un error de un $\pm 6,3 \%$ si se considera un muestreo aleatorio simple.

${ }^{3}$ Los porcentajes alcanzan valores mayores al $100 \%$ ya que se analiza una pregunta de respuesta múltiple. Esto significa que cada asociación (caso) explicitó más de una relación con cada una de las categorías o tipos de contrapartes (respuestas), lo que supone una contabilización de respuestas que supera el número de casos.

${ }^{4}$ Cabe consignar que un $17,9 \%$ de asociaciones no fueron clasificadas dado que en alguna de los componentes alcanzaron puntuaciones de neutralidad, lo que hizo imposible definir su endogamia-exogamia y/o verticalidad y horizontalidad. 
Polis, Revista Latinoamericana, Volumen 14, $N^{\circ}$ 42, 2015

\section{Bibliografía}

Aldridge, S.,Halpern, D. y Fitzpatrick S. (2002), Social Capital: A discussion paper. London: Performance and Innovation Unit.UK.

Aparicio, R. y Tornos A. (2010), Las asociaciones de inmigrantes en España. Una visión de conjunto. Madrid: Ministerio de Trabajo y Asuntos Sociales, Subdirección General de Información y Administraciones Públicas. España.

Berry, J., Phinney, J., Sam, D. y Vedder, P. (2006), Immigrant youth in cultural transition: Acculturation, identity, and adaptation across national contexts. Psychology Press. N. J. EEUU.

Ídem (2006), Immigrant youth in cultural transition: Acculturation, identity and adaptation across national context. New Jersey: Mahwah.

Brazán, J. (2009), “Los refugiados peruanos y sus asociaciones políticas en Santiago de Chile (1990-2006)”. EIAL: Estudios Interdisciplinarios de America Latina y el Caribe, 20(1).

Cachón, L. (2008), “La integración de y con los inmigrantes en España: debates teóricos, políticas y diversidad territorial”. Política y sociedad, 45(1).

Cinalli, M. (2007), “The Impact of 'Relational Structures' upon Collective Action: A Comparison of Unemployment and Asylum in New Labour Britain”. Working Papers - CEVIPOF (23).

Coleman, J. (1990), Foundations of Social Theory. Cambridge (Massachusetts): Belknap Press. EEUU.

del Olmo, N. (2003), “Construcción de identidades colectivas entre inmigrantes: ¿interés, reconocimiento y/o refugio?” Reis 4(104).

Durston, J. (2000), Construyendo capital social comunitario: una experiencia de empoderamiento rural en Guatemala. Naciones Unidas, Comisión Económica para América Latina y el Caribe.

Fullaondo, A. (2007), “La inmigración en España: Una aproximación metropolitana comparada”. Revista ACE (Arquitectura, Ciudad y Entorno) 4.

González, A. y Morales, L. (2006), “Las Asociaciones de Inmigrantes en Madrid: una nota de investigación sobre su grado de integración política”. Revista española del tercer sector (4).

INE- ENI (2011), Informe Encuesta Nacional de Inmigrantes. Madrid: Instituto Nacional de Estadística. España.

Jensen, F. y Valdebenito, X. (2010), “De inclusiones y exclusiones: una 
perspectiva de la inmigración desde la Asociatividad en Chile”. Miradas en Movimiento (3).

Jones, S. (2005), “Community-based ecotourism: The significance of social capital”. Annals of Tourism Research. 32(2).

Krackhardt, D. y Stern, R.(1988), “Informal networks and organizational crises: an experimental simulation”. Social Psychology Quarterly. 51.

Lin, N. (2002), Social Capital: A Theory of Social Structure and Action. Cambridge University Press. UK.

Lozares, C. (2003), “Valores, campos y capitales sociales”. Redes 4(2).

Lozares, C., López-Roldán, P., Pericàs, V., Miquel, J. y Martí Olivé, J. (2011), “Cohesión, Vinculación e Integración sociales en el marco del Capital Social”. Redes 20(1).

Millán, R. y Gordon, S. (2004), “Capital social: una lectura de tres perspectivas clásicas”. Revista mexicana de sociología. 66(4).

Moncusí A. y Rodrigo, M. (2013), “El rol del asociacionismo de inmigrantes africanos en la construcción de cohesión social y la convivencia en Cataluña, Navarra y la Comunidad Valenciana”. Miradas cruzadas. RIS, 71(Extra_1).

Morales, L., Anduiza, E., Rodríguez , E. y San Martín, J. (2008), “Capital social, pautas identitarias y actitudes hacia 'los otros': la incorporación cívica de la población de origen inmigrante en Barcelona y Madrid.” Panorama social (8).

Morell, A. (2005), “El papel de las asociaciones de inmigrantes en la sociedad de acogida: cuestiones teóricas y evidencia empírica”. Migraciones (17).

Ostrom, E., Ahn, T. y Olivares, C. (2003), “Una perspectiva del capital social desde las ciencias sociales: capital social y acción colectiva”. Revista Mexicana de Sociología. 65(1).

Patulny, R. y Svendsen G. (2007), “Exploring the social capital grid: bonding, bridging, qualitative, quantitative”. International Journal of Sociology and Social Policy. 27(1/2).

Penninx, R., y Martiniello, M. (2006), “Procesos de integración y políticas (locales): Estado de la cuestión y algunas enseñanzas”. Revista española de investigaciones sociológicas, 116(1).

Portes, A., y Böröcz, J. (1998), “Migración contemporánea. Perspectivas teóricas sobre sus determinantes y sus modalidades de incorporación”. G. Malgesini (comp.), Cruzando fronteras. Migraciones en el sistema mundial, Icaria, Barcelona. 
Polis, Revista Latinoamericana, Volumen 14, $N^{\circ}$ 42, 2015

Putnam, R. (1995), “Bowling Alone: America’s Declining Social Capital”. Journal of Democracy. 6(1).

Putnam, R. (2001), “La comunidad próspera. El capital social y la vida pública”. Págs. 89-104 en Capital social, Zona Abierta, editado por Francisco Herreros y Andrés de Francisco. Fundación Pablo Iglesias. Madrid, España.

Sarriera, J. (2003), “Estudios actuales sobre la aculturación en latinos: revisión y nuevas perspectivas”. Revista Interamericana de Psicología. 37(2).

Solé, C. y Perella, S.(2005), “Intereses e identidad”. Praxis Sociológica 9(1).

Stefoni, C. (2004), Inmigración y ciudadanía: la formación de comunidades peruanas en Santiago y la emergencia de nuevos ciudadanos. Universidad de Chile. Departamento de Ciencia Política. Instituto de Ciencia Política.

Thayer, E. (2013), “Expectativas de reconocimiento y estrategias de incorporación”. Polis (07176554), (35).

Toral, G. (2010), «Las asociaciones de inmigrantes como sociedad civil: un análisis tridimensional». Revista española de investigaciones sociológicas,132(1).

Torres, O. y Garcés, A. (2013), “Representaciones sociales de migrantes peruanos sobre su proceso de integración en la ciudad de Santiago de Chile”. Polis (Santiago), 12(35).

Uphoff, N. (2003), "El capital social y su capacidad de reducción de la pobreza”. Atria, Raúl; Siles, Marcelo; Arraigada, Irma; Robinson, J. y Whiteford, S. (comps.) Capital social y reducción de la pobreza en América Latina y el Caribe: en busca de un nuevo paradigma. CEPAL.

Veredas, S.(2003), “Las asociaciones de inmigrantes en España: Práctica clientelar y cooptación política”. Revista internacional de sociología (36).

Vranken, J. (2001), “No social cohesion without social exclusion”. EUREX, vol. 5. University of Antwerp. Bélgica.

Woolcock, M. (1998), “Social capital and economic development: Toward a theoretical synthesis and policy framework". Theory and society, 27(2). 